\title{
Comparison of the quality of the result of digitising panoramic analogue films using transmission and reflection scanner mode with direct digital imaging standard
}

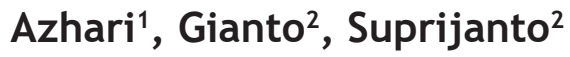 \\ ${ }^{1}$ Department of Dentomaxillofacial Radiology, Faculty of Dentistry Universitas Padjadjaran, \\ Indonesia \\ ${ }^{2}$ Center for Instrumentation Technology and Automation, Faculty of Industrial Technology Bandung \\ Institute of Technology, Indonesia
}

\begin{abstract}
Introduction: The use of panoramic $x$-ray radiographs with analogue film negatives is still widely used. Contrast differences in the radiograph are determined by the level of light transmitted from the viewer to the film negative and visual perception of the radiologist, which can cause differences and errors of interpretation. This study was aimed to compare the quality of scanners produced by the transmission and reflection modes objectively with direct digital imaging standard. Methods: This study used a descriptive comparative design with purposive sampling method. Radiographic analysis was conducted towards 30 analogue radiographs of Radiology laboratory of Universitas Padjadjaran Dental Hospital. Digitisation was performed using a scanner with setting modes (reflection and transmission, 8-bit intensity resolution and spatial resolution of $800 \mathrm{dpi}$ ). Digitising the negative radiographs of analogue films into digital radiographs will get a range of percentage pixels. Histogram of radiographs with a percentage of pixels in the grey level range 0 - 64 exceeds 50\%, including "under-exposed", in the grey level range 192 - 255 exceeding 50\%, including "over-exposed". The evaluation was carried out to observe the radiograph exposure homogeneity by dividing the radiograph into 4 parts. Each part was calculated as the mean pixel value; then the standard deviation was searched for the four mean values (STDMI - standard deviation mean intensity) determined the homogeneity. Results: Digitising type of radiograph reflection was included under-exposed because the percentage of pixels in the grey level range 0 - 64 was $86.94 \%$. STDMI consecutive reflection $=8.03$, transmission $=18.05$, direct digital imaging $=20.86$. Conclusion: Digitisation with scanner transmission mode is the best result objectively because it approaches the quality value of direct digital imaging standard.
\end{abstract}

Keywords: Objective quality, homogeneity, reflection, transmission

P-ISSN 1979-0201, e-ISSN 2549-6212; Available from: http://jurnal.unpad.ac.id/pid/article/view/21155 DOI: 10.24198/pjd.vol31no2.21155

Submission: Dec 26, 2018; Accepted: Jul 17, 2019; Published online: Jul 31, 2019

"Corresponding author: Azhari, Department of Dentomaxillofacial Radiology, Faculty of Dentistry Universitas Padjadjaran, Indonesia. Jalan Sekeloa Selatan I, Bandung, Indonesia, 40132. Phone: +62 813-2277-9900; Email: azhari@fkg.unpad.ac.id 


\section{INTRODUCTION}

The use of conventional panoramic $x$-ray radiographs with negative film analogues is still widely used in dental radiology clinical laboratory in Indonesia nowadays.

In conventional systems for visualisation and interpretation, film negative radiographs are placed on top of a diffuse light source (viewer). The difference in contrast on the radiograph is determined by the level of light transmitted from the viewer to the negative film as well as the visual perception of the radiologist.

The ability of radiologists to distinguish between soft and hard tissue and the interpretation of abnormalities in patients is primarily determined by the range of contrasts visualised on the radiograph.

In addition to conventional devices, Universitas Padjadjaran Dental Hospital Bandung Indonesia uses direct digital imaging panoramic $x$-ray. The advantage compared to visualised equipment is that the final radiograph obtained from the device is already in the DICOM digital radiograph format (digital imaging and communications in medicine).

The digital format makes it possible to make corrections of contrast ranges as needed through radiographic processing. ${ }^{4-6}$ While the contrast quality of conventional devices' film negatives is primarily determined by the film washing process, and there is no chance to make contrast range correction. The contrast range of the film negative radiograph, still varying and different from the contrast range of direct digital imaging radiographs, can give doubt the results of interpretation.

The scanner used in this study is a scanner that can set the reflection and transmission modes as digitising media. The use of this scanner is still rarely done, and the mode selection settings is essential for optimal results.

The radiographic contrast range characteristics of digitisation results will digitise the radiographs resulting from direct digital imaging that will primarily determine as a reference. The purpose of this study was to compare the quality of scanners produced by the transmission and reflection modes objectively with direct digital imaging standard.

\section{METHODS}

This study used a comparative descriptive design with purposive sampling. Samples used were 30 panoramic radiographs from the patients of Universitas Padjadjaran Dental Hospital, then scanned using a reflection scanner and transmission scanner to produce 60 new radiographic data.

All data were processed with Personal Computer (PC) device with Intel Pentium Processor specifications, Dual Core $2 \mathrm{GHz}, 4 \mathrm{~GB}$ RAM; Epson Perfection V700 Scanner, Software, LED LCD Screen, 42LV350, Window XP Professional SP2 operating system, and Epson Scan Program for radiograph processing.

Observation of the brightness level was carried out using histogram analysis of the radiographic contrast range using scanner software. Histogram procedure for digitising negative film samples was performed in the reflection mode setting, 8 bits, spatial $800 \mathrm{dpi}$, as shown in Figure 1.

Homogeneity observation and evaluation were conducted by dividing the radiograph into 4 sections. Each section was being measured for the mean pixel value, and standard deviation mean intensity value (STDMI). Homogeneity analysis was analysed using the standard deviation formula. Standard deviation is a statistically measured data distribution of the pixel grey level of an image. This value represents the contrast level of the image. The standard deviation used was the statistical standard deviation for the population of Karl Pearson. ${ }^{13}$

\section{RESULTS}

All results are presented in the form of image and histogram. Also, data anaysis results are presented in tables. Table 1 shows the comparison of the results of the three radiograph histograms using the reflection, transmission, and Direct Digital Imaging scanner are shown in. While Table 2 and Table 3 consecutively shown a comparative description of the results of the histogram digitisation of the scanner, transmission, and Direct Digital Imaging mode; and comparative description of standard deviation of the mean intensity (STDMI) on 4 radiograph sections. Separated figure presents the histogram results. 


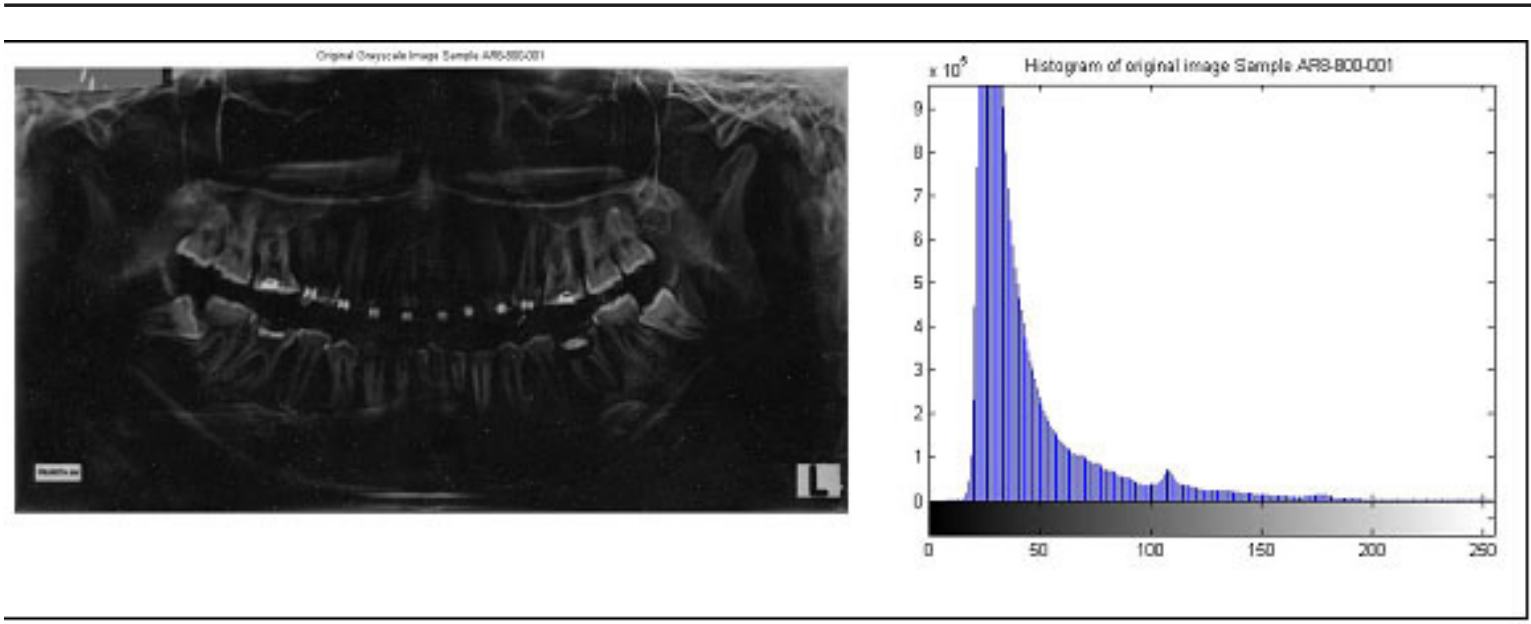

Figure 1. Digitised radiographs and histograms with reflection scanner mode, 8 bits, $800 \mathrm{dpi}$
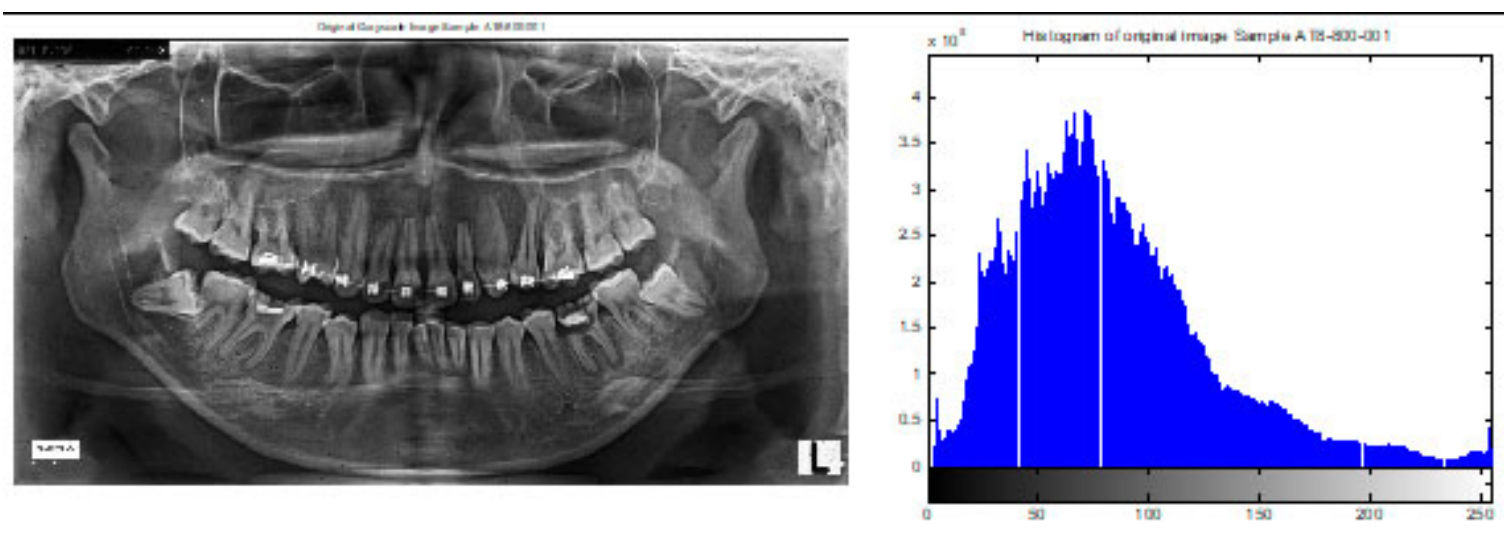

Figure 2. Digitised radiographs and histograms with transmission scanner mode, 8 bits, $800 \mathrm{dpi}$
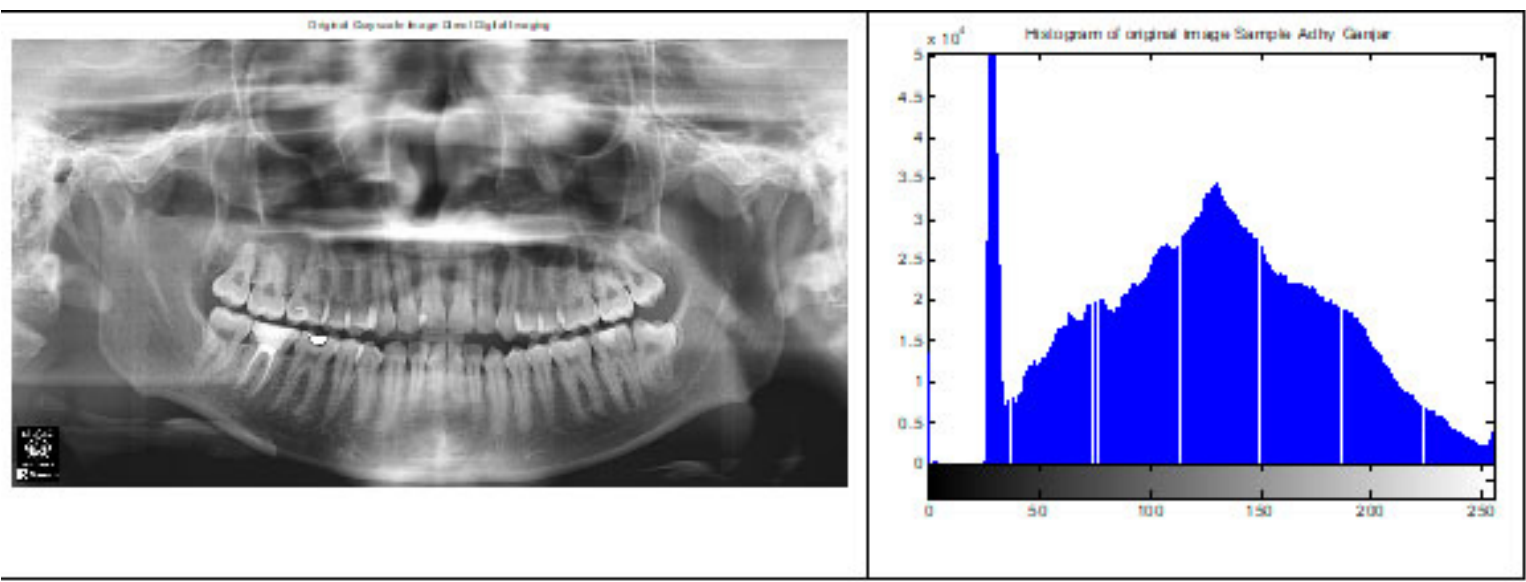

Figure 3. Direct Digital Imaging histogram

Table 1. Digitised radiographic results with spatial resolution of $800 \mathrm{dpi}$

\begin{tabular}{cccc}
\hline Scanner mode & $\begin{array}{c}\text { Average spatial resolution } \\
\text { (pixel) }\end{array}$ & $\begin{array}{c}\text { Average of file size } \\
(\mathbf{k b})\end{array}$ & $\begin{array}{c}\text { Radiographs size } \\
(\mathrm{cm})\end{array}$ \\
\hline Spatial resolution of $\mathbf{8 0 0} \mathbf{d p i}$ & $7969 \times 4266$ & 15.510 & $25.30 \times 13.54$
\end{tabular}




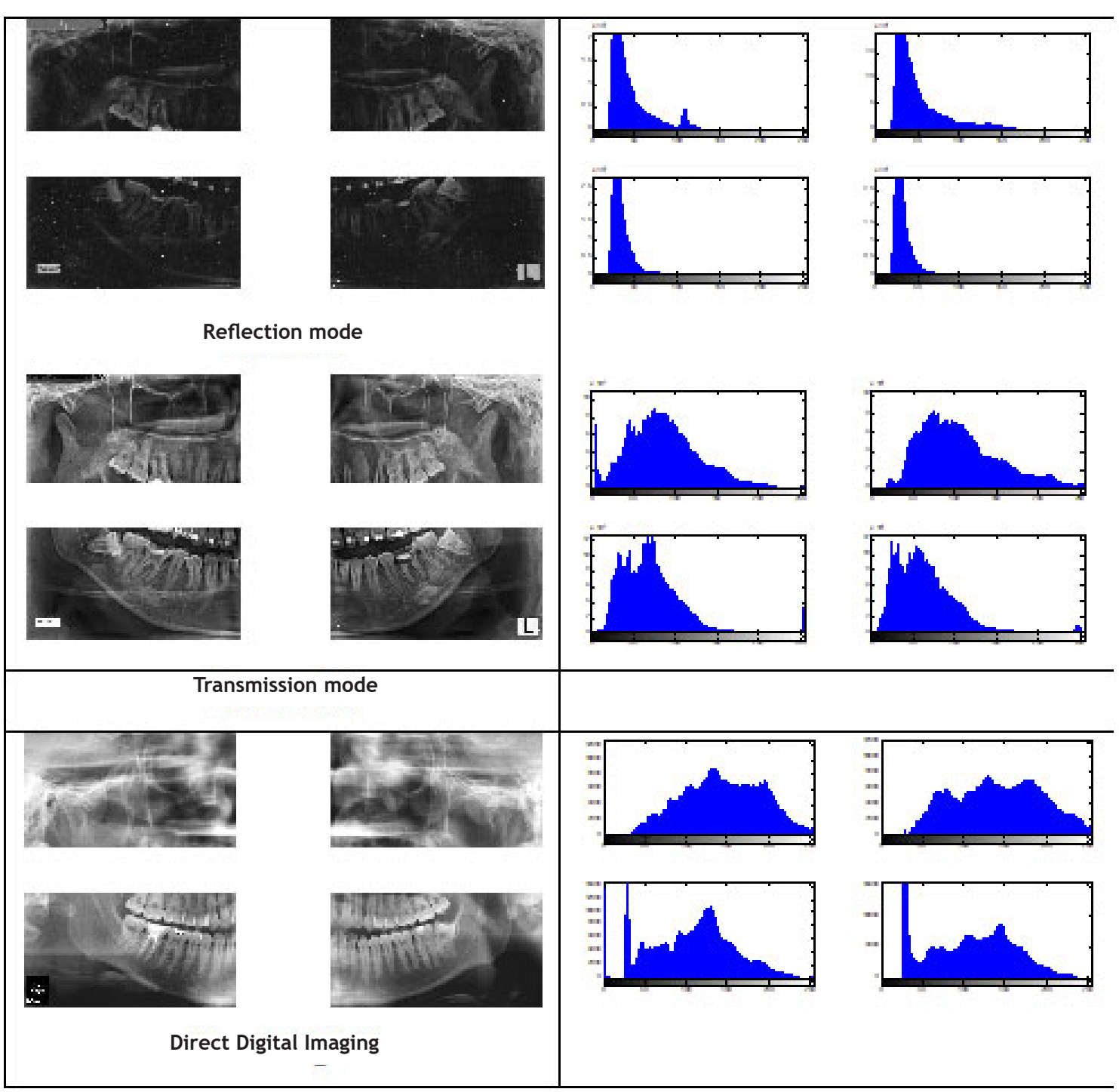

Figure 4. Histogram of 4 radiograph sections with reflection, transmission, and Direct Digital Imaging modes, with resolution intensity of 8 bits, 800 dpi spatial resolution

Table 2. Description of comparison average of digitisation histogram scanner mode reflection, transmission, and Direct Digital Imaging results

\begin{tabular}{lcccc}
\hline Radiograph digitalisation mode & Pixel mean value & Standard deviation & Pixel $<64$ & Pixel $<192$ \\
\hline Reflection/8 bit/ $800 \mathrm{dpi}$ & 41.53 & 27.03 & $86.94 \%$ & $0.08 \%$ \\
Transmission/8 bit/800 dpi & 84.65 & 46.75 & $36.58 \%$ & $3.66 \%$ \\
Direct Digital Imaging & 125.89 & 55.27 & $15.38 \%$ & $12.48 \%$
\end{tabular}

Table 3. Description of standard deviation of the mean intensity (STDMI) on 4 radiograph sections

\begin{tabular}{|c|c|}
\hline Radiograph results & STDMI \\
\hline Reflection & $\mathrm{STDMI}=8.03$ \\
\hline Transmission & $\mathrm{STDMI}=18.05$ \\
\hline Direct Digital & STDMI $=20.86$ \\
\hline
\end{tabular}




\section{DISCUSSION}

An essential initial parameter setting in the digitisation process by a scanner is the dpi (dot per inch) size. The dpi size represents the spatial resolution of a radiograph, which is an exceptional measure of the unequal distribution of the radiograph row and column grid lattices during digitising process. ${ }^{4,5}$

The evaluation value of the resolution measure in this study was $800 \mathrm{dpi}$ spatial. The 800 dpi size indicates that a 1 -inch square radiograph is digitised to $(800 \times 800)$ pixels, meaning that the larger the dpi size, the higher the number of pixels formed on the same radiograph size.

Spatial resolution represents the ability of radiographs to distinguish information that is spatially close together. Table 1 shows the radiographic spatial resolution of $800 \mathrm{dpi}$ size and file size. The higher the spatial resolution of a radiograph, the higher the radiograph's ability to reveal details of an object on the radiograph. The spatial resolution also results in larger radiographic file sizes. The size of the file is important because it is related to the length of the algorithm running process when improving the quality of the radiograph and the need for RAM used. ${ }^{6,7}$

The visual ability of the human eye is also limited in distinguishing radiographically spatially contiguous information. In this study, digitalisation was set with a setting of $800 \mathrm{dpi}$. For panoramic radiograph analysis, the radiographic spatial resolution of the digitisation with the 800 dpi setting is already high enough to be displayed on the monitor.

In addition to spatial resolution setting, the scanner also provides depth resolution options. Depth resolution represents a subtle measure of the roughness of the division of grey level at the time of digitisation. For grayscale types, the Epson Perfection V700 scanner has 8-bit quantisation capabilities. The 8 -bit size indicates that there is a possibility of $28=256$ grey levels on the digitising radiograph, meaning that each pixel on the radiograph has the opportunity to be filled with one grey level value between 0 (black) to 255 (white).

Figure 1 and 2 visually digitising 8-bit resolution radiographs are sufficient to be used for displays on the LCD and LED displays. Meanwhile, as a comparison for evaluating the selection of reflection or transmission mode digitising settings, a radiograph of the results of direct digital imaging was used as shown in Figure 3.

The parameters used in comparing the digitisation radiograph results are the radiographic statistical characteristics of the mean pixel value (mean) and regional standard deviation (standard deviation) of the radiograph. The pixel average represents the radiograph brightness measure, while the regional standard deviation represents the radiographic contrast level measure. Ideal radiographs have an average value $(\mu)$ of between $100-200$ or equivalent to $0.3921-0.7843$ for normalized radiographs $[0 ; 1]$, and the average of the regional standard deviation $(\sigma)$ of between 40 . 80 or equivalent to $0.1568-0.3137$ for normalized radiographs $[0 ; 1]$.

Table 2 shows the statistical characteristics of radiographs resulting from digitisation in the reflection and transmission modes, compared to radiographs resulting from direct digital imaging. Direct digital imaging radiographs have contrast brightness levels in the ideal range $(100<\sigma=125.89$ $<200$ and $40<\sigma=55.27<80$ ), when compared to the two types of digitising systems (reflection and transmission modes), the radiograph results digitisation with the transmission mode is closer to the brightness and contrast of the radiograph results of direct digital imaging. Radiographs of the results of digitisation of the type of reflection have a low pixel mean (by 41.53 from the range 0 255). A low mean pixel value indicates a panoramic radiograph that appears dark.

Also, represented by the histogram graph, the pixels on the radiograph resulting from digitization of the type of reflection are distributed over a narrow grey level range, that is to say, the contrast level is low, corresponding to the minimum standard deviation of the radiograph (27.03). The radiograph results of digitising the type of transmission have a mean pixel value of 84.65.

This value is not yet in the ideal range like radiographs from direct digital imaging. The brightness level is better than radiographs resulting from digitisation of reflection types. Variation in the level of radiographic intensity resulting from digitising the type of transmission 
is higher. That is, the radiographic contrast range is wider and affects the ability of the radiograph to distinguish objects in the radiograph.

Table 2 also shows the percentage of the number of pixels in the grey level range of $0-64$ (1/4 intensity range) and 192 (3/4 intensity range) - 255. These parameters were used to evaluate radiographic intensity imbalances. Radiographs with a percentage of the number of pixels in the grey level range from 0 to 64 exceeding was $50 \%$, including under-exposed, while percentages of the number of pixels in the grey level range $192-255$ exceeded 50\%, including "over-exposed". Thus, radiographs resulting from digitisation of the type of reflection were "under-exposed" because the percentage of the number of pixels in the grey level range of 0 - 64 was $86.94 \%$.

Evaluation of the observation of radiographic exposure homogeneity was performed by dividing the radiograph into 4 sections. Each section was calculated the mean pixel value, then the standard deviation of the four mean values (STDMI - standard deviation mean intensity) was sought. STDMI > 30 indicated non-homogeneous radiographic exposures. Table 3 shows the results of the evaluation of the homogeneity of the radiographically exposed expose.

STDMI of the three dental panoramic digital radiographs showed values of $<30$, meaning that the exposed radiographs were relatively homogeneous. The level of exposed homogeneity of dental panoramic radiographs is influenced by the homogeneity of the scanner's light source during digitisation process and the homogeneity of the x-ray beam at exposure.

The results of the evaluation of the level of brightness and contrast, as well as the imbalance of the intensity of radiographs digitisation with a comparison in the form of direct digital radiographs and transmission types, show more optimal results than the reflection type results.

\section{CONCLUSION}

Digitisation with scanner transmission mode is the best result objectively because it approaches the quality value of direct digital imaging standard.

\section{REFERENCES}

1. White SC, Pharoah MJ. Oral radiology: Principles and interpretation. $7^{\text {th }}$ ed. St. Louis: Mosby-Elsevier; 2014. p. 7.

2. Rios-Santos JV, Ridao-Sacie C, Bullon P, Fernandez-Palacin A, Segura-Egea JJ. Assessment of Periapical Status: A Comparative Study Using Film-Based Periapical Radiographs and Digital Panoramic Images. Med Oral Patol Oral Cir Bucal. 2010; 15(6): e952-6. DOI: 10.4317/medoral.15.e952

3. Lin WC, Wang JW, Lin SY. Contrast Enhancement of X-ray Image Based on Singular Value Selection. Opt Eng. 2010; 49(4): 047005. DOI: 10.1117/1.3378111

4. Morimoto $\mathrm{Y}$, Tanaka T, Yamamoto N, Kodama M, Seta Y, Habu M, et al. New Trends and Advances in Oral and Maxillofacial Imaging. Current Med Imag Rev. 2009; 5(4): 226-37. DOI: $10.2174 / 157340509790112790$

5. Young IT, Gerbrands JJ, Van Vliet LJ. Fundamentals of Image Processing. $2^{\text {nd }}$ ed. Delft: Delft University of Technology; 2012. p. 7.

6. Garg R, Mittal B, Garg S. Histogram Equalization Techniques For Image Enhancement. Int J Electro Comm Tech. 2011; 2(1): 107-11.

7. Salem Saleh Al-amri, N.V. Kalyankar, S.D. Khamitkar, Linear and Non-Linear Contrast Enhancement Image, IJCSNS International Journal of Computer Science and Network Security. 2010;10(2):

8. Wang, Xin;Wong, Brian Stephen;Chen, Guan Tui, Spotting Defects More Clearly: Radiographic Image Enhancement, Industrial Heating.2011;1 : 145-54

9. Papakosta TK, Savva AD, Economopoulos TL, Matsopoulos GK, Gröhndal HG. An automatic panoramic image reconstruction scheme from dental computed tomography images. Dentomaxillofac Radiol. 46 (2017) 1-14.

10. De Oliveira MV, Wenzel A, Campos PS, SpinNeto R. Quality assurance phantoms for cone beam computed tomography: a systematic literature review. Dentomaxillofac Radiol. 46 ;(2017) :52-60. 
11. Singh, Sukhjinder, R.K. Bansal, Savina Bansal, Comparative Study and Implementation of Image Processing Techniques Using MATLAB, International Journal of Advanced Research in Computer Science and Software Engineering. 2012;2(3):125-31

12. Yousif Mohamed Yousif Abdallah, Arwa Ahamed Almoustafa, Gehan Elhadi, Mamdouh
Mohammed, Osman Khalafallah, Tayseer Khalid, Application of analysis approach in Noise Estimation in Panoramic X-rays images using image processing program (MatLab), Canadian Journal on Medicine. 2011;2(3)

13. Pearson K. Contributions to the Mathematical Theory of Evolution. Phil Transact Royal Soc London. 1894; 185: 71-110. 